\title{
ON SOME AGEING PROPERTIES OF GENERAL REPAIR PROCESSES
}

\author{
MAXIM FINKELSTEIN, ${ }^{*}$ University of the Free State and \\ Max Planck Institute for Demographic Research
}

\begin{abstract}
We consider ageing properties of a general repair process. Under certain assumptions we prove that the expectation of an age at the beginning of the next cycle in this process is smaller than the initial age of the previous cycle. Using this reasoning, we show that the sequence of random ages at the start (end) of consecutive cycles is stochastically increasing and is converging to a limiting distribution. We discuss possible applications and interpretations of our results.
\end{abstract}

Keywords: General renewal process; virtual age; degradation; ageing distributions; failure rate; mean remaining lifetime; repairable systems

2000 Mathematics Subject Classification: Primary 60K10

Secondary 60N05

\section{Introduction}

A convenient mathematical description of repair processes uses a concept of stochastic (or failure) intensity (see Aven and Jensen (1999)). Consider, for example, a renewal process (i.e. perfect, instantaneous repair) with an underlying absolutely continuous distribution, $F(t)$, $t \in[0, \infty)$, a failure rate $\lambda(t)$, and a sequence of waiting times $\left\{S_{n}\right\}, \quad n \geq 1$, where $S_{0}=0$. Denote the sequence of independent and identically distributed (i.i.d.) inter-arrival times by $\left\{T_{n}\right\}, n \geq 1$, where $S_{1}=T_{1}$. The stochastic intensity in this case is compactly written via the corresponding indicator function as

$$
\lambda_{t}=\sum_{n=0}^{\infty} \lambda\left(t-S_{n}\right) \mathbf{1}_{\left\{S_{n} \leq t<S_{n+1}\right\}}, \quad t \geq 0 .
$$

Denote by $A_{t}$ the age process, which corresponds to the renewal process (1),

$$
A_{t}=\sum_{n=0}^{\infty}\left(t-S_{n}\right) \mathbf{1}_{\left\{S_{n} \leq t<S_{n+1}\right\}}, \quad t \geq 0 .
$$

Thus, $A_{t}$ starts at $t=0$ as a linear function with a unit slope. It jumps down to 0 at $S_{1}$, which is the time of the first renewal, and so on. The age of a repairable system in this case is just the time elapsed since the last renewal.

As a minimal repair does not change the age of a system, its age process is trivial, i.e.

$$
A_{t}=t, \quad t \geq 0 .
$$

Received 20 April 2006; revision received 13 March 2007.

* Postal address: Department of Mathematical Statistics, University of the Free State, PO Box 339, 9300 Bloemfontein, South Africa. Email address: finkelm.sci@ufs.ac.za 
Assume now that a repair action at $t=t_{1}$ (i.e. the realization of $T_{1}$ ) decreases the age of a system not to 0 , as in the case of a perfect repair, but to $v_{1}=q t_{1}, \quad 0<q<1$, and the system starts the second cycle with this initial age in accordance with the cumulative distribution function $1-\bar{F}\left(v_{1}+t\right) / \bar{F}\left(v_{1}\right)$, where $\bar{F} \equiv 1-F$. This age is often called the virtual age. For convenience, we will omit the term 'virtual' in what follows. The forthcoming cycles are defined in a similar way to form a process of general repair (see Kijima (1989), Stadje and Zuckerman (1991), Finkelstein (1992), (2000), Baxter et al. (1996), and Last and Szekli (1998), to name a few). The sequence of ages after the $i$ th repair $\left\{V_{i}\right\}_{i \geq 0}$ in this model, for a specific case of a linear, deterministic repair function $q t$, is defined as

$$
\begin{aligned}
V_{0} & =0, \\
V_{1} & =q T_{1}, \\
V_{2} & =q\left(V_{1}+T_{2}\right), \\
& \vdots \\
V_{i} & =q\left(V_{i-1}+T_{i}\right),
\end{aligned}
$$

and distributions of the corresponding inter-arrival times for realizations $v_{i}$ are given by

$$
\bar{F}_{i}(t)=\frac{\bar{F}\left(v_{i-1}+t\right)}{\bar{F}\left(v_{i-1}\right)}, \quad i \geq 1 .
$$

Therefore, the age process for this model is

$$
A_{t}=\sum_{n=0}^{\infty}\left(t-S_{n}+V_{n}\right) \mathbf{1}_{\left\{S_{n} \leq t<S_{n+1}\right\}}, \quad t \geq 0 .
$$

Other settings and generalizations can be also considered (see, for example, Last and Szekli (1998) for relevant examples). All these models have a crucial common feature: the corresponding age processes are defined by the generic distribution $F(t)$ and only the 'position' of the starting point of each cycle (as, for example, in (3)) depends on the concrete model.

Define a stochastic point process as stochastically ageing, if its inter-arrival times $\left\{T_{n}\right\}$, $n \geq 1$, are stochastically decreasing, i.e.

$$
T_{i+1} \leq_{\mathrm{st}} T_{i}, \quad i \geq 1,
$$

Thus, the renewal process is not ageing in this sense, whereas the nonhomogeneous Poisson process is ageing if its rate is an increasing function.

The following definition deals with ageing properties of the sequence of ages at the start (end) of cycles for the point processes of the described types.

Definition 1. The age process is called stochastically increasing if the (embedded) sequence of ages at the start (end) of cycles is stochastically increasing.

If, for example, a generic $F(t)$ is of the increasing failure rate (IFR) type, then the stochastically increasing age process describes overall deterioration of our repairable system with time, which is the case for various wearing out systems in practice.

In what follows we will study the properties of the age process (4) with a nonlinear quality of repair function $q(t)$. Under rather weak assumptions it will be shown that this process is 
stochastically increasing and is becoming stable in distribution (i.e. converges to a limiting distribution as $t \rightarrow \infty)$. These issues for the linear $q(t)$ were first addressed in Finkelstein (1992), where the corresponding renewal-type equations were also derived. The rigorous and detailed treatment of monotonicity and stability for rather general age processes driven by generic $F(t)$ was given by Last and Szekli (1998). The approach of Last and Szekli was based on applying some fundamental probabilistic results: a Loynes-type scheme and Harrisrecurrent Markov chains were used. Our approach for a more specific model (but with weaker assumptions on $F(t)$ and with a time dependent $q(t))$ is based on a direct probabilistic reasoning and on the appealing 'geometrical' notion of an equilibrium age $v^{*}$.

Apart from obvious engineering applications, these results may have some important biological interpretation. Most biological theories of ageing agree that the process of ageing can be considered as some process of 'wear and tear' (see, for example, Yashin et al. (2000) ). The existence of repair mechanisms in organisms decreasing the accumulated damage on various levels is also a well-established fact. As in the case of DNA mutations in the process of cell replication, this repair is not perfect. Asymptotic stability of the repair process means that an organism, as a repairable system, is practically not ageing in the defined sense for sufficiently large $t$. Therefore, the deceleration of human mortality rate at advanced ages (see, for example, Thatcher (1999)) and even the approaching of this rate to the mortality plateau can be explained in this way. As we will see, this conclusion relies on an important assumption that a repair action decreases the overall accumulated damage and not only its last increment. It is worth noting that another possible explanation of mortality deceleration phenomenon at advanced ages is via the concept of population heterogeneity (see Finkelstein and Esaulova (2006) for mathematical details).

\section{The quality of the repair function}

Assume now that a linear function $q t$ in (2) is now an increasing, continuous in $[0, \infty)$, and concave function $q(t), q(0)=0$. Therefore,

$$
q\left(t_{1}+t_{2}\right) \leq q\left(t_{1}\right)+q\left(t_{2}\right), \quad t_{1}, t_{2} \in[0, \infty) .
$$

Also, let

$$
q(t)<q_{0} t,
$$

where $q_{0}<1$, which shows that repair rejuvenates the failed item to some extent and that $q(t)$ cannot be arbitrarily close to $q(t)=t$ (minimal repair).

Let a cycle start with an age $v$. Denote by $T(v)$ the cycle duration with the corresponding survival function given by the right-hand side of (3) for $v_{i-1}=v$. The next cycle will start at a random age $q(v+T(v))$. In this section we will be interested in some equilibrium age $v^{*}$. We define this age as a solution to the following equation:

$$
\mathrm{E}[q(v+T(v))]=v .
$$

Thus, if some cycle of a general repair process starts at age $v^{*}$, then the next cycle will start with a random age with expectation $v^{*}$, which is obviously a martingale property.

Theorem 1. Let $\left\{T_{n}\right\}, \quad n \geq 1$, be a process of general repair with an increasing, continuous quality of repair function $q(t)$, defined by (5) and (6).

Assume that the generic distribution $F(t)$ has a finite first moment and that the corresponding failure rate is either bounded from below for the sufficiently large t by $c>0$ or is converging 
to 0 as $t \rightarrow \infty$ such that

$$
\lim _{t \rightarrow \infty} t \lambda(t)=\infty
$$

Then there exists at least one solution to (7) and, if there is more than one, the set of these solutions is bounded in $[0, \infty)$.

Proof. (a) As $\mathrm{E}[T(0)]<\infty$, it is evident that $\mathrm{E}[T(v)]<\infty, v>0$. If $\lambda(t)$ is bounded from below by $c>0$, then

$$
\mathrm{E}[T(v)] \leq \frac{1}{c}
$$

therefore, applying (5), we obtain

$$
\mathrm{E}[q(v+T(v)] \leq q(v)+\mathrm{E}[T(v)] .
$$

It follows from (6) and (9) that

$$
\mathrm{E}[q(v+T(v))]<v,
$$

for sufficiently large $v$. On the other hand, $\mathrm{E}[q(T(0))]>0$, which proves the first part of the theorem, as the function $\mathrm{E}[q(v+T(v))]-v$ is continuous in $v$, positive at $v=0$, and negative for sufficiently large $v$.

(b) Now, let $\lambda(t) \rightarrow 0$ as $t \rightarrow \infty$. Consider the following quotient:

$$
\frac{\mathrm{E}[T(v)]}{v}=\frac{\int_{v}^{\infty} \exp \left(-\int_{0}^{x} \lambda(u) \mathrm{d} u\right) \mathrm{d} x}{v \exp \left(-\int_{0}^{v} \lambda(u) \mathrm{d} u\right)} .
$$

Applying l'Hôpital's rule and using the assumption (8), we obtain

$$
\lim _{v \rightarrow \infty} \frac{\mathrm{E}[T(v)]}{v}=\lim _{t \rightarrow \infty} \frac{1}{\lambda(v) v-1}=0 .
$$

Therefore, applying (9) and taking into account (6) and (10), we obtain

$$
\frac{\mathrm{E}[q(v+T(v))]}{v} \leq \frac{q(v)}{v}+\frac{\mathrm{E}[T(v)]}{v}<1 .
$$

The last inequality holds for sufficiently large $v$. Using the same argument as in the first part of the proof completes our reasoning.

Corollary 1. If $F(t)$ is IFR then the conditions of Theorem 1 hold and there is, at least, one solution to (7).

Remark 1. The sufficient condition (8) is a rather weak one stating, in fact, that $t \lambda(t)$ must just have a limit as $t \rightarrow \infty$, which should not be finite. For instance, the 'bizarre' failure rate $\lambda(t)=|\sin t| \ln t / t$, which tends to 0 as $t \rightarrow \infty$, does not comply with (8). On the other hand, it is clear that, for example, for the Weibull distribution with decreasing failure rate (8) holds.

Theorem 2. Let $F(t)$ be IFR. Assume that a current cycle of a general repair process starts at age $v^{*}+\Delta v$, where $v^{*}$ is an equilibrium solution to (7) and $\Delta v>0$.

Then the expectation of an age at the start of the next cycle 'will be closer' to $v^{*}$, i.e.

$$
v^{*}<\mathrm{E}\left[q\left(v^{*}+\Delta v+T\left(v^{*}+\Delta v\right)\right)\right]<v^{*}+\Delta v
$$


Proof. As stated in Corollary 1, at least one solution to (7) exists in this case. Let us first prove the second inequality in (11). Taking into account that $q(t)$ is an increasing function and that the random variables $T(v)$ are stochastically decreasing in $v$ (for increasing $\lambda(t)$ ), we have

$$
\mathrm{E}\left[q\left(v^{*}+\Delta v+T\left(v^{*}+\Delta v\right)\right)\right]<\mathrm{E}\left[q\left(v^{*}+\Delta v+T\left(v^{*}\right)\right)\right] .
$$

When obtaining this inequality the following simple fact was used. If two distributions are ordered as $\bar{F}_{1}(t)>\bar{F}_{2}(t), t \in(0, \infty)$, and $g(t)$ is an increasing function, then by integrating by parts it is easy to see that

$$
\int_{0}^{\infty} g(t) \mathrm{d} F_{2}(t)<\int_{0}^{\infty} g(t) \mathrm{d} F_{1}(t) .
$$

Finally,

$$
\mathrm{E}\left[q\left(v^{*}+\Delta v+T\left(v^{*}\right)\right)\right] \leq \mathrm{E}\left[q\left(v^{*}+T\left(v^{*}\right)\right)\right]+q(\Delta v)=v^{*}+q(\Delta v)<v^{*}+\Delta v .
$$

The first inequality in (11) is proved using similar arguments. We have

$$
\mathrm{E}\left[q\left(v^{*}+\Delta v+T\left(v^{*}+\Delta v\right)\right)\right]>\mathrm{E}\left[q\left(v^{*}+T\left(v^{*}\right)\right)\right]=v^{*},
$$

after observing that the random variable $v^{*}+\Delta v+T\left(v^{*}+\Delta v\right)$ is stochastically larger than $v^{*}+T\left(v^{*}\right)$.

Corollary 2. If $F(x)$ is IFR, then (7) has a unique solution.

Proof. Assume that there are two solutions to (7), i.e.

$$
\begin{aligned}
\mathrm{E}\left[q\left(v^{*}+T\left(v^{*}\right)\right)\right] & =v^{*} \\
\mathrm{E}[q(\tilde{v}+T(\tilde{v})] & =\tilde{v} .
\end{aligned}
$$

Let $\tilde{v}=v^{*}+\Delta v, \Delta v>0$. Then, in accordance with (11), we obtain

$$
\mathrm{E}[q(\tilde{v}+T(\tilde{v}))]=\mathrm{E}\left[q\left(v^{*}+\Delta v+T\left(v^{*}+\Delta v\right)\right)\right]<v^{*}+\Delta v=\tilde{v},
$$

which contradicts (13).

Remark 2. When (7) has a unique solution, it can be shown, similarly to the proof of (11), that

$$
v^{*}-\Delta v<\mathrm{E}\left[q\left(v^{*}-\Delta v+T\left(v^{*}-\Delta v\right)\right)\right]<v^{*} .
$$

Remark 3. The results of this section hold when repair action is stochastic. That is, $\left\{Q_{i}\right\}$, $i \geq 1$, is a sequence of i.i.d. random variables (independent from other stochastic components of the model) with support in $[0,1]$ and $\mathrm{E}\left[Q_{i}\right]<1$.

The described properties show that there is a shift in the direction of the equilibrium point $v^{*}$ of the starting age of the next cycle as compared with the starting age of the current cycle. Note that for the minimal repair process the corresponding shift is always in the direction of infinity. 


\section{Monotonicity and stability of an age process}

Denote the age distribution at the start of the $(i+1)$ th cycle by $\theta_{i+1}^{\mathrm{S}}(v), \quad i=1,2, \ldots$, and denote the corresponding age distribution at the end of the previous $i$ th cycle by $\theta_{i}^{\mathrm{E}}(v)$, $i=1,2, \ldots$ It is clear that, in accordance with our model (see (2)-(3) and (5)-(6)), we have

$$
\theta_{i+1}^{\mathrm{S}}(v)=\theta_{i}^{\mathrm{E}}\left(q^{-1}(v)\right), \quad i=1,2, \ldots,
$$

where the inverse function $q^{-1}(v)$ is also increasing. This can be easily seen, since

$$
\theta_{i+1}^{\mathrm{S}}(v)=\mathrm{P}\left(V_{i+1}^{\mathrm{S}} \leq v\right)=\mathrm{P}\left(q\left(V_{i}^{\mathrm{E}}\right) \leq v\right)=\mathrm{P}\left(V_{i}^{\mathrm{E}} \leq q^{-1}(v)\right),
$$

where $V_{i+1}^{\mathrm{S}}$ and $V_{i}^{\mathrm{E}}$ are random ages at the start of $(i+1)$ th cycle and at the end of the previous one respectively. The following theorem states that the age processes under consideration are stochastically increasing.

Theorem 3. Random ages at the end (start) of each cycle in the general repair model (2)-(3) and (5)-(6) form the following stochastically increasing sequences:

$$
\bar{\theta}_{i+1}^{\mathrm{E}}(v)>\bar{\theta}_{i}^{\mathrm{E}}(v), \quad \bar{\theta}_{i+2}^{\mathrm{S}}(v)>\bar{\theta}_{i+1}^{\mathrm{S}}(v), \quad v>0, i=1,2, \ldots
$$

Proof. We shall prove the first inequality; the second one follows trivially from (14). Consider the first two cycles. Let $v_{1}^{\mathrm{E}}$ be the realization of $T_{1}$, where $T_{1}$ is the age at the end of the first cycle and at the same time the duration of this cycle. Then (for this realization) the age at the end of the second cycle is $q\left(v_{1}^{\mathrm{E}}\right)+T\left(q\left(v_{1}^{\mathrm{E}}\right)\right)$. It is clear that this random variable is stochastically larger than $T_{1}$ and, as this property holds for each realization, (15) holds for $i=1$.

Assume that (15) holds for $i=n-1, n \geq 3$. Due to definition of age at the start and the end of a cycle, integrating by parts and using (14), we obtain

$$
\begin{aligned}
\theta_{n}^{\mathrm{E}}(v) & =\int_{0}^{v}\left(1-\exp \left(-\int_{x}^{v} \lambda(u) \mathrm{d} u\right)\right) \mathrm{d}\left(\theta_{n}^{\mathrm{S}}(x)\right) \\
& =\int_{0}^{v} \theta_{n}^{\mathrm{S}}(x) \mathrm{d}_{x}\left(\exp \left(-\int_{x}^{v} \lambda(u) \mathrm{d} u\right)\right) \\
& =\int_{0}^{v} \theta_{n-1}^{\mathrm{E}}\left(q^{-1}(x)\right) \mathrm{d}_{x}\left(\exp \left(-\int_{x}^{v} \lambda(u) \mathrm{d} u\right)\right), \\
\theta_{n+1}^{\mathrm{E}}(v) & =\int_{0}^{v}\left(1-\exp \left(-\int_{x}^{v} \lambda(u) \mathrm{d} u\right)\right) \mathrm{d}\left(\theta_{n+1}^{\mathrm{S}}(x)\right) \\
& =\int_{0}^{v} \theta_{n+1}^{\mathrm{S}}(x) \mathrm{d}_{x}\left(\exp \left(-\int_{x}^{v} \lambda(u) \mathrm{d} u\right)\right) \\
& =\int_{0}^{v} \theta_{n}^{\mathrm{E}}\left(q^{-1}(x)\right) \mathrm{d}_{x}\left(\exp \left(-\int_{x}^{v} \lambda(u) \mathrm{d} u\right)\right),
\end{aligned}
$$

where we use the fact that $\exp \left(-\int_{x}^{x+(v-x)} \lambda(u) \mathrm{d} u\right)=\exp \left(-\int_{x}^{v} \lambda(u) \mathrm{d} u\right)$ is the probability of survival from initial age $x$ to age $v>x$. This can also be interpreted via the remaining lifetime concept.

Taking into account the induction assumption and comparing (16) and (17), using similar reasoning to that used while obtaining (12), we have

$$
\theta_{n}^{\mathrm{E}}(v)<\theta_{n-1}^{\mathrm{E}}(v) \Longrightarrow \theta_{n}^{\mathrm{E}}\left(q^{-1}(v)\right)<\theta_{n-1}^{\mathrm{E}}\left(q^{-1}(v)\right) \Longrightarrow \theta_{n+1}^{\mathrm{E}}(v)<\theta_{n}^{\mathrm{E}}(v),
$$

which completes the proof. 
The next theorem states that the increasing sequences of survival functions $\bar{\theta}_{i}^{\mathrm{E}}(v), \bar{\theta}_{i}^{\mathrm{S}}(v)$ are converging to a limiting survival function as $i \rightarrow \infty$, and that the decreasing sequences of distribution functions $1-\bar{\theta}_{i}^{\mathrm{E}}(v), 1-\bar{\theta}_{i}^{\mathrm{S}}(v)$ are converging to a limiting distribution function as $i \rightarrow \infty$. Thus, the repair process is stable in the defined sense.

Theorem 4. Let the governing distribution $F(t)$ in a general repair model (2)-(3) and (5)-(6) be IFR.

Then there exists the following limiting distributions for ages at the start and at the end of cycles:

$$
\lim _{i \rightarrow \infty} \theta_{i}^{\mathrm{E}}(v)=\theta_{\mathrm{L}}^{\mathrm{E}}(v) \text { and } \lim _{i \rightarrow \infty} \theta_{i}^{\mathrm{S}}(v)=\theta_{\mathrm{L}}^{\mathrm{S}}(v) .
$$

Proof. The proof is based on Theorems 2 and 3. As the sequences (15) are increasing at each $v>0$, there can be only two possibilities. Either there are limiting distributions (18) with uniform convergence in $[0, \infty)$ or the ages grow infinitely, as for the case of minimal repair $(q=1)$. The latter means that, for each fixed $v>0$,

$$
\lim _{i \rightarrow \infty} \theta_{i}^{\mathrm{E}}(v)=0 \text { and } \lim _{i \rightarrow \infty} \theta_{i}^{\mathrm{S}}(v)=0 .
$$

Assume that (19) holds and consider the sequence of ages at the start of a cycle. Then, for an arbitrarily small $\zeta>0$, we can find $n$ such that

$$
\mathrm{P}\left(V_{i}^{\mathrm{S}} \leq v^{*}\right) \leq \zeta, \quad i \geq n,
$$

where $v^{*}$ is an equilibrium point which is unique and finite according to Corollary 2. It follows from (11) that, for each realization $v_{i}^{\mathrm{S}}>v^{*}$, the expectation of the starting age at the next cycle is smaller than $v_{i}^{\mathrm{S}}$. On the other hand, the 'contribution' of ages in $\left[0, v^{*}\right)$ can be made arbitrarily small if (19) holds. Therefore, it can be easily seen that for sufficiently large $i$

$$
\mathrm{E}\left[V_{i+1}^{\mathrm{S}}\right]<\mathrm{E}\left[V_{i}^{\mathrm{S}}\right] .
$$

This inequality contradicts Theorem 3, according to which expectations of ages form an increasing sequence. Therefore assumption (19) is wrong, and the limiting property (18) holds. As previously, the result for the second limit in (18) follows trivially from (14).

Corollary 3. The sequence of inter-arrival lifetimes $\left\{T_{n}\right\}, n \geq 1$, is stochastically decreasing to a random variable with a limiting distribution, i.e.

$$
\lim _{i \rightarrow \infty} F_{i}(t)=F_{\mathrm{L}}(t)=\int_{0}^{\infty}\left(1-\exp \left(-\int_{v}^{v+t} \lambda(u) \mathrm{d} u\right)\right) \mathrm{d}\left(\theta_{\mathrm{L}}^{\mathrm{S}}(v)\right) .
$$

Proof. Equation (20) follows immediately after taking into account that convergence in (18) is uniform. On the other hand, comparing

$$
F_{i}(t)=\int_{0}^{\infty}\left(1-\exp \left(-\int_{v}^{v+t} \lambda(u) \mathrm{d} u\right)\right) \mathrm{d}\left(\theta_{i}^{\mathrm{S}}(v)\right)
$$

and

$$
F_{i+1}(t)=\int_{0}^{\infty}\left(1-\exp \left(-\int_{v}^{v+t} \lambda(u) \mathrm{d} u\right)\right) \mathrm{d}\left(\theta_{i+1}^{\mathrm{S}}(v)\right),
$$

it is easy to see, using the same argument as in the proof of Theorem 2, that $F_{i+1}(t)>F_{i}(t)$, $t>0, i=1,2, \ldots$, (i.e. a stochastically decreasing sequence of inter-arrival times), as $\theta_{i+1}(v)<\theta_{i}(v)$, and the integrand function is increasing in $v$ for the IFR case. 


\section{Concluding remarks}

Under reasonable assumptions, we show that the general repair process with a quality of repair function $q(t)$, defined by (5) and (6), is stochastically increasing. Therefore, this property describes a certain overall deterioration of a repairable object. On the other hand, Theorem 4 states that this deterioration slows down and eventually vanishes at infinity, which means that the defined type of repair is decreasing age (i.e. wear) in a way that is 'sufficient' for this result.

Model (2) is usually referred to in the literature as the Kijima-2 general repair model. It is worth noting that it was independently suggested and analyzed in Finkelstein (1988). Unfortunately this paper was not translated into English.

\section{Acknowledgement}

The author would like to thank two anonymous referees, whose suggestions helped to improve the presentation of this paper.

\section{References}

Aven, T. And Jensen, U. (1999). Stochastic Models in Reliability (Appl. Math. (New York) 41). Springer, New York. Baxter, L. A., KiJima, M. and Tortorella, M. (1996). A point process model for the reliability of the maintained system subject to general repair. Stoch. Models 12, 37-65.

Finkelstein, M. S. (1988). Engineering systems with imperfect repair. Nadejnost i Control Kachestva 8, 7-12 (in Russian).

FinKelsteIn, M. S. (1992). A restoration process with dependent cycles. Automatic Remote Control 53, 115-1120.

Finkelstein, M. S. (2000). Modeling a process of non-ideal repair. In Recent Advances in Reliability Theory, eds N. Limnios and M. Nikulin, Birkhäuser, Boston, MA, pp. 41-53.

Finkelstein, M. and Esaulova, V. (2006). Asymptotic behavior of a general class of mixture failure rates. Adv. Appl. Prob. 38, 244-262.

KiJima, M. (1989). Some results for repairable systems with general repair. J. Appl. Prob. 26, 89-102.

LAST, G. AND SzeKLI, R. (1998). Asymptotic and monotonicity properties of some repairable systems. Adv. Appl. Prob. 30, 1089-1110.

Stadje, W. And Zuckerman, D. (1991). Optimal maintenance strategies for repairable systems with general degree of repair. J. Appl. Prob. 28, 384-396.

Thatcher, A. R. (1999). The long-term pattern of adult mortality and the highest attained age. J. R. Statist. Soc. A $162,5-43$.

Yashin, A., Iachine, I. A. And Begun, A. S. (2000). Mortality modeling: a review. Math. Population Studies 8, 305-332. 